\title{
Serology: A Precise Tool in Diagnosis and Epidemiology of COVID-19
}

\section{Bramhadev Pattnaik ${ }^{1}$, Mahendra P Yadav ${ }^{2}$, Sharanagouda Patil ${ }^{3 *}$ and Pinaki Panigrahi ${ }^{4}$}

${ }^{1}$ One Health Center for Surveillance and Disease Dynamics, AIPH University,

Bhubaneswar, Odisha and Former Director, ICAR-Directorate of Foot and Mouth

Disease, Mukteswar, India

${ }^{2}$ Former Vice-Chancellor, SVP University of Agriculture and Technology, Meerut, India

${ }^{3}$ ICAR-National Institute of Veterinary Epidemiology and Disease Informatics

(NIVEDI), Bengaluru, India

${ }^{4}$ Department of Pediatrics, Division of Neonatal-Perinatal Medicine, Georgetown

University Medical Center, Washington, D.C. USA

*Corresponding Author: Sharanagouda Patil, ICAR-National Institute of

Veterinary Epidemiology and Disease Informatics (NIVEDI), Bengaluru, India.
Received: June 01, 2020

Published: August 26, 2020

(C) All rights are reserved by Sharanagouda

Patil., et al.

\section{Abstract}

The coronavirus infectious disease-2019 (COVID-19), caused by a $\beta$-Coronavirus, named SARS-CoV-2, has become a global pandemic since its origin in Wuhan, China during the last week of December 2019, affecting 212 countries and territories in the World involving all the five continents. Prompt and precise diagnosis of the disease is central to its control and eradication. Real- time polymerase chain reaction (real-time PCR) using dual labelled TaqMan probe and targeting two genomic areas, usually RdRp and envelope (E) regions, of the virus is being extensively used for the diagnosis of SARS-CoV-2 in respiratory clinical specimens. As stage of the infection cannot be ascertained during collection of respiratory specimens for nucleic acid test (NAT; RT-PCR), this may lead to false negatives (error of omission) as virus load in the respiratory exudates and saliva gradually decreases with the increase in time post infection. Virus excretion would be maximum during clinical sickness that follows incubation period of usually up to $\sim 14$ days and clinical samples collected during this period are suitable for PCR diagnosis than those collected after clinical sickness. In addition, there are other variables, like quality of swabs and virus transport medium, PCR protocol and reagents, enzyme inhibitors, and proficiency of the manpower engaged in executing diagnostic techniques may affect the quality of the test result. There are three grades of clinical sickness in COVID-19, viz. asymptomatic, mild symptomatic and highly symptomatic. Available data indicate that about $50 \%$ of the people exposed to SARS-CoV-2 infection may become asymptomatic, as was observed in case of the COVID-19 affected Japan cruise ship 'Diamond Princess' with 3,711 people on board. In case of asymptomatic and mild symptomatic cases, due to low virus load in the clinical specimens collected, the negative result in NAT/PCR need to be cross checked using a suitable antibody assay. It is known that virus load in the body and the quantum of virus excreted in body fluids gradually decreases with the remission from sickness, whereas quantum of specific antibody against the virus increases with time till plateau. Anti-virus antibody remains in the host for longer duration and can be detected even after clearance of the infection from the body. Therefore, NAT must be complemented by antibody test to enhance quality of diagnosis and mitigate errors of omission. Further, unlike NAT/PCR, serology/antibody test is a powerful tool in tracking virus transmission, estimating actual number of cases, and epidemiological mapping of the disease in a population. Further, availability of a precise antibody test system/assay would be handy for post-pandemic surveillance of COVID-19. The current review includes the results of COVID-19 diagnosis and kinetics of antibody response reported by different authors/groups of scientists that vouch for quick development of a 'COVID-19 antibody assay' system for use in epidemiological studies of the disease.

Keywords: Antibody Test; COVID-19; Epidemiology; PCR; SARS-CoV-2; Serology 


\section{Introduction}

SARS-CoV-2 causes an acute viral infection in humans with a median incubation period of 3 days [1]. Coronaviruses (CoVs) are single-stranded positive sense RNA viruses that exist in four genetic types, viz., $\alpha$-coronavirus, $\beta$-coronavirus, $\delta$-coronavirus and $\gamma$-coronavirus (ICTV). Genetic analysis revealed that SARS-CoV-2 is a Beta-coronavirus (genus) and genetically clusters with in the subgenus Sarbecovirus (lineage B), together with some bat virus strains with $>96 \%$ genetic identity. A total of seven CoVs have been identified causing mild to severe disease in humans; 04 with seasonal circulation causing mild cold (HKU1, NL63, OC43 and 229E), and the remaining 03 are zoonotic ones, viz., SARS-CoV (2003), MERS-CoV (2012) and SARS-CoV-2 (2019), that originated in different bat species and transmitted to human through an intermediate host; Civet in case of SARS-CoV, Dromedary Camel in case of MERS-CoV, and possibly Pangolin [2] in case of SARS-CoV-2 that is having about 79\% genetic similarity with SARS-CoV and only $50 \%$ with MERS-CoV. Structural modelling revealed that SARSCoV-2 binds to ACE2 with more than 10-fold higher affinity than SARS-CoV, that explains faster transmissibility of SARS-CoV-2 in humans compared to SARS-CoV, and also higher number of confirmed cases of COVID-19 compared with SARS-CoV Infection [3]. The basic reproduction number (Ro) of COVID-19 vary from 2-3.3 that also explains its higher transmissibility compared to SARS and MERS [4,5]. Accordingly, as on 24 May 2020, 05:31 GMT, there were 28,15,429 active cases of COVID-19 in the World involving 213 countries and territories covering all the five continents (https://www.worldometers.info/coronavirus/). As on this date and time, number of active cases in the USA, Brazil, Russia, Spain and India were 1666828, 349113, 335882, 282370 and 73610, respectively. This reveals active transmission of the virus even after prompt diagnosis employing nucleic acid tests and implementation of social distancing and lockdowns. The necessity of the time is to investigate sero-epidemiology of the disease/infection at the earliest employing appropriate antibody tests in order map the population(s) exposed to the virus irrespective of outcome of the infection. Further, NAT negative people need to be confirmed by antibody assay(s) in order to reduce possible spread of the virus infection by such persons. Antibody assays employing different viral antigens like RdRp, nucleoprotein, S1 protein, receptor binding domain (RBD) are being used in limited scales in different countries, other than India, to identify and diagnose infected people. The present review compiles reported results of antibody assay vis-à-vis RT-PCR in diagnosis of SARS-CoV-2 infection.

\section{Coronavirus and cellular infectivity}

Coronaviruses (CoV) are a large family of positive sense single stranded RNA viruses that cause illness ranging from the com- mon cold to more severe diseases such as Severe Acute Respiratory Syndrome (SARS-CoV of 2003), Middle East Respiratory Syndrome (MERS-CoV of 2012), and Coronavirus Infectious Disease-2019 (COVID-19) in human beings. Although SARS-CoV and MERS-CoV infections have a higher mortality rate than COVID-19, the SARSCoV-2 spreads much faster than the former two diseases. Since 1930 , the CoVs of different strains are known to infect and cause disease in poultry, bovines, porcine, canine and feline species. The SARS-CoV-2 is a novel coronavirus which has not previously been detected in humans, and has higher transmission rate than the earlier two CoV. Coronavirus has only 4 structural proteins: the spike (S), membrane (M), envelope (E), and nucleocapsid (N) proteins. The transmembrane glycoprotein spikes (S protein) of $\mathrm{CoV}$ are highly immunogenic and are target of immune response. The receptor binding domain (RBD) in the S protein is particularly targeted by neutralizing antibodies. The receptor binding motif (RBM) with in the RBD region of both SARS-CoV and SARS-CoV-2 play major role in virus neutralization and the similarity in the amino acid residues between the $\mathrm{RBM}$ of both the viruses is limited to only 59\%; also there are neutralizing epitopes outside the RBM [6]. The $S$ glycoprotein on the viral surface is trimeric and mediates entry of virus into host cells. The $\mathrm{S}$ protein has two functional subunits that mediate cell attachment (the S1 subunit, consisting of four domains S1A through S1D) and fusion of the viral and cellular membrane (the S2 subunit) required for endocytosis. The spike proteins of SARS-CoV-2 with 1,273 residues, (strain Wuhan-Hu-1) and that of SARS-CoV with 1,255 residues, (strain Urbani) are $77.5 \%$ identical in amino acid sequence and structurally similar, and bind to the cellular receptor through S1B domain. Receptor interaction causes irreversible conformational changes in the spike proteins resulting in membrane fusion for endocytosis [7]. The $\mathrm{S}$ protein determines host tropism and transmissibility of the virus. The RBD of both SARS-CoV-2 of 2019 and SARS-CoV of 2003 recognize and bind to angiotensin converting enzyme 2 (ACE2) receptor on the susceptible cells, whereas that of MERS-CoV binds to DPP4 (dipeptidyl peptidase 4) receptor [8,9]. As a whole, SARS-CoV-2 is genetically distinct from both SARS-CoV of 2003 and MERS-CoV of 2012 [10].

\section{COVID-19 testing}

COVID-19 testing includes methods that detect the presence of (i) virus genome by reverse -transcription- polymerase chain reaction (RT-PCR) or loop mediated isothermal nucleic acid amplification (LAMP), and (ii) antibodies produced in response to infection. Detection of antibodies can be used both for diagnosis of the disease and population surveillance. Antibody tests show how many people are exposed to the infection, and also identify mildly symptomatic and asymptomatic cases. Accurate estimation of case fatality/mortality rate (CFR/CMR) of the disease and the level of 
herd immunity in the population can be determined only from the results of antibody detection in serological survey. However, the duration of immune response and immunity to COVID-19 is yet to be known, as the disease has started only since December 2019. In the absence of a suitable antibody assay system, only RT-PCR is now being used in the diagnosis of COVID-19 using respiratory specimens.

\section{Polymerase chain reaction (PCR)}

Polymerase chain reaction (PCR) is a process that amplifies a defined segment of DNA to be detected. The SARS-CoV-2 being a RNA virus, reverse transcription- polymerase chain reaction (RTPCR) and its several modifications including Real Time- RT-PCR (quantitative PCR), and its further modifications like Syber green assay that measures the temperature melting (Tm) of the amplicon and TaqMan assay that uses a dual-labelled probe in addition to 2 primers, are being used in diagnosis on nasopharyngeal swabs, sputum and saliva. The likelihood of detecting the virus in the collected clinical specimen depends on how much time has passed since the person was infected. In one study, a positive test result was highest at week 1 (100\%), followed by $89.3 \%, 66.1 \%, 32.1 \%$, $5.4 \%$ and $0 \%$ at weeks 2, 3, 4, 5 and 6, respectively (SymptomBased Strategy to Discontinue Isolation for Persons with COVID-19 (Centers for Disease Control and Prevention, USA., 30 April 2020; Profile of RT-PCR for SARS-CoV-2: a preliminary study from 56 COVID-19 patients. Clinical Infectious Diseases. 19 April 2020. doi:10.1093/cid/ciaa460/5822175). This genome detection kinetics is the limitation of RT-PCR as compared to serology for antibody detection for the diagnosis of COVID-19, and can lead to error of omission. In a cohort study comprising of sixty-seven COVID-19 patients, the median duration of SARS-CoV-2 RNA shedding were 12 (3-38), 19 (5-37), and 18 (7-26) days in nasopharyngeal swabs, sputum and stools, respectively. Only 13 urine (5.6\%) and 12 plasma (5.7\%) samples were virus positive [11]. Another study [12,13] showed that RT-PCR-based viral RNA detection is sensitive and can effectively confirm early SARS-CoV-2 infection. A cohort study [14] conducted at two hospitals in Hong Kong during January-February 2020 that included 23 laboratory- confirmed COVID-19 patients (median age 62 years [range 37 - 75]) revealed the median viral load in posterior oropharyngeal saliva or other respiratory specimens at $5 \cdot 2 \log _{10}$ copies per ml. Virus load in saliva was the highest during the first week after onset of symptoms and subsequently declined with time. In one patient, viral RNA was detected 25 days after onset of symptom. Older age was correlated with higher viral load. Quantum of virus load in the specimen determines the result of PCR test.

\section{Seroconversion and its detection}

Since the beginning of COVID-19, reverse transcriptase poly- merase chain reaction (RT-PCR) has routinely been used for its diagnosis. However, several authors have pointed out the poor performance of this technique, particularly in terms of sensitivity $[10,15]$; sensitivity of RT-PCR could be as low as 38\% [16]. Serology, for detection of anti-viral IgM/IgG, has been used as a complementary assay to RT-PCR $[17,18]$. RT-PCR detects only the virus genome, whereas antibody tests are useful in checking the community spread as it indicates exposure to the virus, and the isotype of antibody (IgG/IgM) detected, speaks about the time of virus infection. As per the WHO, seroconversion is the transition from the status of seronegative (no antibodies in the serum, or present but below the limit of detection) to seropositive in which antibodies can be detected in serum samples. Isotype switching, also called immunoglobulin class switching, is the conversion of production of antibodies by B cells from one type to another. IgM isotype antibodies are the first to be generated against an antigen, then the isotype switches to IgG antibodies, which are more effective in immune protection. The antibody isotype(s) present in a patient serum/ specimen can give important information about the timing of initial exposure to the virus, as well as provide information on the progression of the disease and prognosis. IgM indicates fresh infection, and IgG indicates past infection or convalescence. Virus-specific antibody detection is important for (1) diagnosis of suspected cases with negative RT-PCR result, (2) detection of asymptomatic infection, and (3) tracking virus transmission and sero-surveillance in the target population to understand virus circulation $[12,13]$.

In the case of the SARS-CoV outbreak (2003-04), serology was primarily an epidemiological tool that could help determine the number of silent infections, progression of the disease, virus transmission patterns, and the possible origin of the virus [19]. Antibody testing for SARS-CoV-2 is at increased demand in order to better quantify the number of cases of COVID-19, including those that may be asymptomatic or have recovered (FDA, USA). Serology tests can identify whether people have been exposed to a particular pathogen by looking at their immune response. In contrast, the RT-PCR tests currently being used globally for diagnosis of COVID-19 can only indicate the presence of viral genome during infection and will not indicate if a person was infected and subsequently recovered. The antibody tests can give greater detail into the prevalence of a disease in a population by identifying individuals who have developed antibodies to the virus [20]. Early in the infection, antibodies may not be detected, and this limits effectiveness of serological assays for diagnosis of COVID-19 [20]. However, serological tests can play a critical role in identifying individuals who have overcome an infection in the past and have developed an immune response. Due to limited serological testing, no country has reliable data on the prevalence of the virus in their population. Serological testing may be helpful for the diagnosis of suspected patients with nega- 
tive RT-PCR results and also for the identification of asymptomatic infections [12,13]. Confirming suspected COVID-19 cases as early as possible with the help of serological testing could reduce risk of exposure during repeated sampling and save valuable RT-PCR tests $[12,13]$. In this study, seven cases with no symptoms and negative RT-PCR result were positive for IgG and/or IgM antibodies, that highlights the importance of serological testing to achieve more accurate estimates of the extent of the COVID19 pandemic.

First time a human monoclonal antibody (MAb 47D11) that neutralizes SARS-CoV-2 and SARS-CoV was reported [21]. The MAb 47D11 binds to a conserved epitope on the RBD (receptor binding domain in spike protein) and neutralizes both SARS-CoV and SARS-CoV-2, through a mechanism that is independent of ACE2 receptor binding inhibition. This MAb will be useful for development of antigen detection tests and serological assays targeting SARS-CoV-2.

\section{Diagnostic significance of Immunoglobulin isotypes}

- IgM antibodies develop early in the infection.

- IgG antibodies develop later, and is the most common isotype of antibody in the blood and other body fluids. The IgG antibodies confer protection against infection and also have memory in the immune system.

IgA antibodies are associated with local/mucosal immunity, and are found on the mucous membranes of the lungs, sinuses, stomach, and intestines. They are also found in saliva and tears, as well as in the blood.

\section{Types of serology assays}

- Rapid diagnostic test (RDT): This is a qualitative (positive or negative) lateral flow assay that is used for detection of antibodies (IgG and IgM), or viral antigen. Available test systems detect IgM/IgG antibodies against nuceloprotein (N/NP) of SARS-CoV-2.

- Enzyme-linked immunosorbent assay (ELISA): This test can be qualitative or quantitative and can make use of whole blood, plasma, or serum samples from patients. Antibodies (IgM/IgG) against spike (S) (either S1 and S2 as a whole or RBD), $\mathrm{N}$ and $\mathrm{M}$ can be detected.

- Neutralization assay: This test detects antibodies in serum/ plasma effective against the virus to clear the infection. There are several modifications of this test.

- Chemiluminescent immunoassay: This test is quantitative and can detect multiple types of immunoglobulins including IgG, IgM, and IgA in whole blood, plasma, and serum.

\section{Detection of viral antigen}

A specific viral antigen can be detected by ELISA. The problem with antigen detection system is that often there could be not enough antigen present in the nasal swab to be detectable; particularly in asymptomatic people. Unlike the RT-PCR test, there is no amplification method for viral proteins in an antigen test. According to the WHO, the sensitivity of antigen detection tests for respiratory diseases like the flu ranges between $34 \%$ and $80 \%$, and accordingly half or more of COVID-19 infected patients could be missed out by such tests, leading to error of omission. However, in some studies the virus could be detected in saliva of $91.7 \%(11 / 12)$ of patients [14].

\section{Kinetics of antibody detection}

IgG antibodies to SARS-CoV-2 generally become detectable 1014 days after infection, and usually peak around 28 days following infection. IgM antibodies may be detected earlier. Since antibodies take time to develop, they are not the best markers of acute infection, but as they may persist in the bloodstream for many years, they are ideal for detecting past infections/convalescence. In a cohort study comprising of 67 COVID-19 patients [11], the anti-N/NP IgM could be detected on day 7 and on day 28 , while IgG was on day 10 and peaked on day 49 after onset of illness. The titres of IgM and IgG were significantly higher in severe patients compared to nonsevere patients $(p<0.05)$. The duration and nature of immunity against SARS-CoV-2 infection is not yet known. The median time to detection of antibodies was similar for SARS-CoV-1 (12 days; IQR 8-15.2 days) and SARS-CoV-2 (11 days; IQR 7.25-14 days), but longer for MERS-CoV (16 days; IQR 13-19 days) [22]. There was no detectable cross-neutralization by SARS patient sera against SARSCoV-2 [23].

A study on acute antibody responses to SARS-CoV-2 in 285 patients with COVID-19 revealed that, $100 \%$ of patients tested positive for antiviral immunoglobulin-G (IgG) within 19 days after symptom onset $[12,13]$. IgG and IgM titres in the severe group were higher than those in the non-severe group. Serological testing may be helpful for the diagnosis of suspected patients with negative RT-PCR results and for the identification of asymptomatic infections. Seroconversion for IgG and IgM occurred simultaneously or sequentially, and both IgG and IgM titres plateaued within 6 days after seroconversion (loc. cit.).

In serum samples available from 16 patients for 14 days or longer after onset of symptom, rate of seropositivity was $94 \%$ for antiNP IgG, 88\% for anti-NP IgM, 100\% for anti-RBD IgG, and 94\% for anti-RBD IgM $[14,24]$. An increase was noted in IgG or IgM antibody levels against NP/RBD in most patients at 10 days or later after on- 
set of symptom. More patients had earlier seropositivity for antiRBD than anti-NP.

The generation of IgG antibody in female patients was stronger than male patients in early phase of the disease [25]. While the underlying mechanisms are not known, this discrepancy in IgG antibody level between male and female patients may potentially lead to negative clinical outcome of COVID-19 in male patients.

\section{Serological assays}

In the case of SARS-CoV outbreak of 2003, serology was primarily used as an epidemiological tool that could help determine inapparent infections, process of disease progression, virus transmission pattern, and the possible origin of the virus [19]. Investigation revealed that COVID-19 patients had IgM sero-reactivity by day 4 post symptom onset, which peaked by day 9 , whereas IgG sharply increased 12 days after onset of symptom, and all viral nucleic acid positive patients were positive for IgG 30 days post symptom onset [18]. In the patients suspected for COVID-19 and tested negative for viral genome, IgM antibodies were detected in $87.5 \%$ and IgG in $70.8 \%$ cases. They showed sensitivity for diagnosis of COVID-19 was $77.3 \%$ for IgM with specificity of $100 \%$ as against $88.3 \%$ and $95 \%$ for IgG, respectively, In the case of COVID-19 diagnosis employing virus genome detection technique, the pre-analytical variables like inconsistency in obtaining nasopharyngeal swabs, the different swabs and transport medium used, time and temperature of specimen transport, and possible presence of nucleic acid/PCR inhibitors in the sample etc can influence the test result [26]. Analysis of serological data may be useful in examining exposure to the virus, but serology can be more challenging to interpret patients with acute infection; cross reactivity with other coronaviruses and pathogens could be a problem $[19,26]$. Coupling the potential limitations and strengths of both viral genome detection and serological assays can increase the rate of diagnosis of COVID-19 infected patients [18]. This investigation (loc. cit.) is a first step towards better understanding of the antibody response elicited against SARS-CoV-2 and provides important insight into the possible characteristics and use of serological tests in COVID-19 pandemic.

Using CHO cell expressed full length SARS-CoV-2 S1 protein as capturing antigen, a SARS-CoV-2 S1 serology ELISA kit was developed. The specificity (means negative as negative) and sensitivity (means positive as positive) of this ELISA was $97.5 \%$ and $97.1 \%$, respectively, with overall accuracy rate of $97.3 \%$ [27]. The assay was able to detect SARS-CoV-2 antibody on day one after the onset of the disease, and it could detect specific antibodies in 28 out of 276 asymptomatic people and one out of five PCR-negative close contacts of COVID-19 patient.
The presence of IgM would suggest recent infection, while IgM negative and IgG positive would suggest a previous infection. This testing strategy would be most effective 1-2 weeks after the initial onset of symptoms and would also help to assess herd immunity and the risk of a new infection for those returning from quarantine. The antibody testing sensitivity ranged from $28.7 \%$ (symptom onset 1-7 days) to $73.3 \%$ (symptom onset 8-14 days), and that of $94.3 \%$ at more than 15 days of symptom onset [28]. Molecular tests have limited sensitivity during the first 7 days of symptom onset (ranging from 67-72\%) which may be due to low viral load early in the disease course or differences in collection technique [28].

Using RBD-IgG ELISA as a screening test for SARS-CoV-2 antibody, followed by confirmation using plaque reduction neutralization test was adapted for large-scale sero-epidemiology studies to assess infection attack rates in the population and define disease severity and herd immunity [29,30]. A positive RBD ELISA result was indicative of prior infection with SARS-CoV-2. Large-scale sero-epidemiological studies can provide population infection attack rates in near real time [31].

Recent studies have evaluated the potential role of IgM antibodies against SARS-CoV-2 as a diagnostic marker of recent infection [32]. Using an ELISA, employing recombinant NP antigen of SARSCoV-2, it was shown that IgM antibodies were detectable in $85 \%$ of COVID-19 confirmed patients at 1-7 days post onset of symptom [33]. These authors stated that though molecular testing remains preferred, with higher sensitivity, during the first 5.5 days after onset of illness, targeting IgM may be useful in suspected covid-19 patients diagnosed negative by molecular methods. The IgM antibodies against the RBD located in the S1 subunit of the spike glycoprotein of the virus could be detected only in about $28 \%$ of patients by day 7 of post symptom onset, whereas $73 \%$ turned out to be positive by day 14 [34]. Similar to IgM, recent studies showed that IgA antibodies against the virus were detectable as early as one day after onset of symptoms [33]. In contrast to IgM and IgA isotypes, detection of IgG antibodies against the virus may have a larger role to play during the COVID-19 pandemic. Further, IgG response is long lasting similar to IgA, and is associated with viral neutralizing activity, that is essential for recovery from the disease [35,36]. Serologic testing to detect IgG isotype antibodies against the virus will play an essential role in determining the true prevalence of this virus [32]. Studies have also suggested fairly high specificity (>95\%) for IgG-based serologic assays for COVID-19 [12,13,32,37]. Data suggested that IgG developed against different SARS-CoV-2 antigens were detectable in patients after at least 8 days post clinical sickness, and over $90 \%$ of patients were seropositive post day 14 of illness, whereas some individuals may take longer time to 
become seropositive depending on their immune status, or may never seroconvert if significantly immunosuppressed [12,13,34].

From prior immunity studies in recovered SARS-CoV patients, neutralizing antibodies were detectable in $89 \%$ of patients up to 2 years after infection, whereas IgG antibodies were undetectable at 6 years $[38,39]$. We have to wait till that time to have similar data in respect of SARS-CoV-2. The rate of asymptomatic infection with COVID-19 has been reported at 4 to $80 \%$ across different populations and exposure scenarios, and therefore, seroprevalence studies will help in establishing a more accurate estimate of the number of infected individuals that in turn will help in determining the true case fatality rate (CFR) at regional, national and global level [40-42].

Serological tests determine what percentage of the population has been exposed to the virus. Early studies suggested that the detection of IgM and IgG in COVID-19 patients typically occurs between 7- 11 days post exposure. Heat inactivation of blood samples at $56{ }^{\circ} \mathrm{C}$ for $30 \mathrm{~min}$ did not affect the results of immunochromatography and chemiluminescent immunoassay for detection of SARS-COV-2 antibodies but can reduce the risk of infection for the laboratory personnel handling the tests [43].

\section{Asymptomatic cases}

On 5 February 2020, a cruise ship, the 'Diamond Princess' hosting 3,711 people was put on quarantine for 2 weeks after a passenger going ashore was diagnosed with COVID-19. Until 20 February 2020, 634 persons on board were tested positive for SARS-CoV-2, out of which 306 were symptomatic and the remaining 328 were asymptomatic (50.5\%) [3]. There are two classes of asymptomatic cases with SARS-CoV-2 infection, viz., (1) people with little or mild symptoms within the incubation period but with onset of symptoms during the quarantine period, and (2) people with no symptoms all the time but tested positive for viral nucleic acid or antibodies. Asymptomatic infection is of concern, and asymptomatic people are called super spreaders. Asymptomatic carriers are the people with mild or no symptoms but positive for viral nucleic acid of SARS-CoV-2 or positive for specific IgM antibody in serum [3]. There are evidences suggesting possible transmission of SARS-CoV-2 from asymptomatic cases. The viral load detected in the asymptomatic patients was similar to that in the symptomatic patients, suggesting the transmission potential of asymptomatic or minimally symptomatic patients [44]. An asymptomatic case tested positive for the virus in faeces, but not in nasopharyngeal swabs, suggests a theoretical possibility of transmission via faecaloral route [45]. Asymptomatic suspected patients should be quarantined and monitored for 14 days, and their quarantine will end when two consecutive samples collected at more than 24 hours interval are negative in nucleic acid test [46]. Application of antibody tests is suitable in screening different age groups of people to determine the number of people infected with little or no symptoms, and estimating the actual number of confirmed cases. With the existence of disagreement in the actual number of asymptomatic cases and their infectivity, larger observational and longitudinal studies using serological tests are needed to elucidate [31]. However, strict quarantine of asymptomatic patients is of great importance in the control of COVID-19 pandemic across the globe, and if various feasible measures are taken to control the transmission, then the outbreak could end quickly and in an effective manner [3].

\section{Host immune response assay}

Myxovirus resistance protein $\mathrm{A}(\mathrm{MxA})$ is a biomarker for viral infection, with low basal concentration (less than $15 \mathrm{ng} / \mathrm{ml}$ ), long half-life (2.3 days) and fast induction (1-2 hours) [47]. It was demonstrated that MxA mRNA is detectable in peripheral blood within 1-2 hours of white blood cells stimulated with interferon (IFN) alpha, and then MxA protein begins to accumulate [48]. In case of MERS-CoV and SARS-CoV, it was demonstrated that these coronaviruses can increase MxA expression in the blood [49]. Many studies have shown that MxA protein expression in peripheral blood is a sensitive and specific marker of viral infection. Expression of MxA protein is regulated exclusively by type I IFNs [47,50]. The MxA gene is expressed in blood mononuclear cells or locally in tissues, and apparently the MxA gene does not respond to other cytokines such as IL-1 or TNF- $\alpha$ [51].

\section{Recombinant viral proteins for use in ELISA}

Now that the genome sequence of SARS-CoV-2 is known, the viral protein(s) of interest can be produced as recombinant protein in E. coli or Eukaryotic/Baculovirus systems in large quantities for use in ELISA. After the antigen is directly bound to the wells, test human serum is added, and a secondary labelled (usually with enzyme HRP) antibody is added that will react to human antibodies (in test serum) bound to the antigen, and the presence of the label (HRP etc.) in the secondary antibody will create a colorimetric or fluorometric output that can be quantified.

\section{Conclusion}

Application of precise antibody assay systems is a must to complement COVID-19 diagnosis by RT-PCR, as there are chances of false negatives (in PCR tests) due to variability in virus load in the clinical materials collected for diagnosis. It is difficult to ensure the stage of infection during collection of clinical samples for nucleic acid tests. Virus load in respiratory exudates is likely to be maximum during clinical sickness, and gradually reduces with remission from the sickness. Therefore, samples collected late in the 
infection may turn out to be negative in nucleic acid tests. However, antibodies elicited following virus infection can be detected for longer periods, and therefore, asymptomatic cases can also be diagnosed by application of antibody tests. Unlike NAT, serology is useful in understanding the spread of the virus and epidemiology of the disease.

\section{Acknowledgements}

Information available in the Internet (NCBI-PubMed, WHO, OIE, ICMR, ICAR, Govt of India, Wikipedia, Newspaper and News periodicals etc) have been incorporated in the present review. We sincerely acknowledge each of these.

\section{Conflict of Interest}

No conflict of interest is declared.

\section{Bibliography}

1. Guan W J., et al. "Clinical characteristics of 2019 novel coronavirus infection in China". The New England Journal of Medicine 382 (2020): 1708-1720.

2. Xiao K., et al. "Isolation of SARS-CoV-2-related coronavirus from Malayan pangolins". Nature (2020).

3. Wang Y., et al. "Asymptomatic Cases with SARS-CoV-2 Infection". Journal of Medical Virology (2020).

4. Majumder M A M and Kenneth D. "Early transmissibility assessment of a novel coronavirus in Wuhan, China”. SSRN (2020).

5. Wu J T., et al. "Nowcasting and forecasting the potential domestic and international spread of the 2019-nCoV outbreak originating in Wuhan, China: a modelling study". Lancet 395.10225 (2020a): 689-697.

6. Chen W H., et al. "Potential for developing a SARS-COV receptor-binding domain (RBD) recombinant protein as a heterologous human vaccine against coronavirus infectious disease (COVID)- 19". Human Vaccines and Immunotherapeutics 16.6 (2020): 1239-1242.

7. Walls A C., et al. "Unexpected receptor functional mimicry elucidates activation of coronavirus fusion". Cell 176 (2019): 1026-1039.

8. Hirano T and Murakami M. "COVID-19: A new virus, but a familiar receptor and cytokinereleasesyndrome". Immunity 52 (2020): 731-733.

9. Sahu KK., et al. "COVID-19: update on epidemiology, disease spread and management". Monaldi Archives for Chest Disease 90.1292 (2020): 197-205.
10. Liu R., et al. "Positive rate of RT-PCR detection of SARS-CoV-2 infection in 4880 cases from one hospital in Wuhan, China, from Jan to Feb 2020". Clinica Chimica Acta 505 (2020): 172-175.

11. Wenting Tan., et al. "Viral Kinetics and Antibody Responses in Patients with COVID-19". medRxiv (2020).

12. Long Q X., et al. "Antibody responses to SARS-CoV-2 in COVID-19 patients: the perspective application of serological tests in clinical practice". medRxiv (2020a).

13. Long Q X., et al. "Antibody responses to SARS-CoV-2 in patients with COVID-19". Nature Medicine (2020b).

14. Kelvin Kai-Wang To., et al. "Temporal profiles of viral load in posterior oropharyngeal saliva samples and serum antibody responses during infection by SARS-CoV-2: an observational cohort study". The Lancet Infectious diseases 20.5 (2020a): 565574.

15. Xie J., et al. "Characteristics of Patients with Coronavirus Disease (COVID-19) Confirmed using an IgM-IgG Antibody Test". Journal of Medical Virology (2020).

16. Liu K., et al. "Clinical features of COVID-19 in elderly patients: A comparison with young and middle-aged patients". Journal of Infection 80.6 (2020): e14-e18.

17. Cassaniti I., et al. "Performance of VivaDiag COVID-19 IgM/IgG Rapid Test is inadequate for diagnosis of COVID-19 in acute patients referring to emergency room department". Journal of Medical Virology (2020).

18. Xiang F., et al. "Antibody detection and dynamic characteristics in patients with COVID-19". Clinical Infectious Diseases (2020): ciaa461.

19. Meyer B., et al. "Serological assays for emerging coronaviruses: Challenges and pitfalls". Virus Research 194 (2014): 175-183.

20. FDA, USA (2020).

21. Wang C., et al. "A Human Monoclonal Antibody Blocking SARSCoV-2 Infection". Nature Communication 11.1 (2020): 2251.

22. Huang A T., et al. "A systematic review of antibody mediated immunity to coronaviruses: antibody kinetics, correlates of protection, and association of antibody responses with severity of disease". medRxiv (2020). 
23. Anderson DE., et al. "Lack of cross-neutralization by SARS patient sera towards SARS-CoV-2". Emerging Microbes and Infections (2020).

24. Kelvin Kai-Wang To., et al. "Consistent Detection of 2019 Novel Coronavirus in Saliva". Clinical Infectious Diseases 71.15 (2020b): 841-843.

25. Zeng F., et al. "A comparison study of SARS-CoV-2 IgG antibody between male and female COVID-19 patients: a possible reason underlying different outcome between sex". Journal of Medical Virology (2020).

26. Stowell S and Guarner J. "Role of serology in the COVID-19 pandemic". Clinical Infectious Diseases (2020).

27. Zhao R., et al. "Early detection of SARS-CoV-2 antibodies in COVID-19 patients as a serologic marker of infection". Clinical Infectious Diseases (2020).

28. Pulia M S., et al. "Multi-Tiered Screening and Diagnosis Strategy for COVID-19: A Model for Sustainable Testing Capacity in Response to Pandemic". Annuals of Medicine 14 (2020): 1-8.

29. Perera R A., et al. "Serological Assays for Severe Acute Respiratory Syndrome Coronavirus 2 (SARS-CoV-2), March 2020". Euro Surveillance 25.16 (2020): 2000421.

30. Wong J Y., et al. "Case fatality risk of influenza A (H1N1pdm09): a systematic review". Epidemiology 24.6 (2013): 830-841.

31. Wu J T., et al. "Inferring influenza infection attack rate from seroprevalence data”. PLoS Pathogens 10.4 (2014): e1004054.

32. Theel E S., et al. "The Role of antibody Testing for SARS-Co: Is There One?". Journal of Clinical Microbiology (2020).

33. Guo L., et al. "Profiling Early Humoral Response to Diagnose Novel Coronavirus Disease (COVID-19)". Clinical Infectious Diseases (2020).

34. Zhao J., et al. "Antibody responses to SARS-CoV-2 in patients of novel coronavirus disease 2019". Clinical Infectious Diseases (2020).

35. Schlomchik M., et al. "The distribution and functions of immunoglobulin isotypes". In: Immunobiology: The Immune System in Health and Disease, 5th ed. Garland Publishing, New York, NY.
36. Casadevall A and Pirofski L A. "The convalescent sera option for containing COVID-19". Journal of Clinical Investigation 130 (2020): 1545-1548.

37. Amanat F., et al. "A serological assay to detect SARS-CoV-2 seroconversion in humans". medRxiv (2020).

38. Tang F., et al. "Lack of peripheral memory B cell responses in recovered patients with severe acute respiratory syndrome: a six-year follow-up study". Journal of Immunology 186 (2011): 7264-7268.

39. Rokni M., et al. "Immune responses and pathogenesis of SARSCoV-2 342 during an outbreak in Iran: Comparison with SARS and MERS". Reviews in Medical Virology 30.3 (2020): e2107.

40. Kimball A., et al. "Asymptomatic and Presymptomatic SARSCoV-2 Infections in Residents of a Long-Term Care Skilled Nursing Facility - King County, Washington, March 2020". Morbidity and Mortality Weekly Report (MMWR) 69 (2020): 377-381.

41. Mizumoto K., et al. "Estimating the asymptomatic proportion of coronavirus disease 2019 (COVID-19) cases on board the Diamond Princess cruise ship, 361 Yokohama, Japan, 2020". Euro Surveillance 25.10 (2020): 2000180.

42. Zhou X., et al. "Follow-up of asymptomatic patients with SARSCoV-2 infection". Clinical Microbiology Infection 26.7 (2020): 957-959.

43. Xue X., et al. "Effect of heat inactivation of blood samples on the efficacy of three detection methods of SARS-CoV-2 antibodies". Nan Fang Yi Ke Da Xue Xue Bao 40.3 (2020): 316-320.

44. Zou L., et al. "SARS-CoV-2 Viral Load in Upper Respiratory Specimens of Infected Patients". The New England Journal of Medicine 382 (2020): 1177-1179.

45. Jiang X., et al. "Asymptomatic SARS-CoV-2 infected case with viral detection positive in stool but negative in nasopharyngeal samples lasts for 42 days". Journal of Medical Virology (2020).

46. Protocols (2020) http://en.nhc.gov.cn/2020-03/29/c_78468. htm.

47. Ronni T., et al. "Control of IFN-inducible MxA gene expression in human cells". Journal of Immunology 150.5 (1993): 1715-1726.

48. Ronni T., et al. "Regulation of IFN-alpha/beta, MxA, 2', 5'-oligoadenylate synthetase, and HLA gene expression in influenza A-infected human lung epithelial cells". Journal of Immunology 158.5 (1997): 2363-2374. 
49. Tynell J., et al. "Middle East respiratory syndrome coronavirus shows poor replication but significant induction of antiviral responses in human monocyte-derived macrophages and dendritic cells". Journal of General Virology 97.2 (2016): 344-355.

50. Staeheli P and Sutcliffe J G. "Identification of a second interferon-regulated murine Mx gene". Molecular and Cellular Biology 8 (1988): 4524-4528.

51. Simon A., et al. "Interferon-regulated Mx genes are not responsive to interleukin-1, tumor necrosis factor, and other cytokines". Journal of Virology 2 (1991): 968-971.

\section{Assets from publication with us}

- Prompt Acknowledgement after receiving the article

- Thorough Double blinded peer review

- Rapid Publication

- Issue of Publication Certificate

- High visibility of your Published work

Website: www.actascientific.com/

Submit Article: www.actascientific.com/submission.php

Email us: editor@actascientific.com

Contact us: +919182824667 